\title{
The Perceptions and Self-management of Depressive Symptoms in Men: A Qualitative Study in a Province of Northeastern Thailand
}

\author{
Netchanok Kaewjanta ${ }^{1}$ and Somporn Rungreangkulkij ${ }^{2, *}$ \\ ${ }^{I}$ Philosophy Program in Nursing Science, Faculty of Nursing, Khon Kaen University, \\ Khon Kaen 40002, Thailand \\ ${ }^{2}$ Centre for Research and Training on Gender and Women's Health, Faculty of Nursing, \\ Khon Kaen University, Khon Kaen 40002, Thailand
}

('Corresponding author's e-mail: somrun@kku.ac.th)

Received: 23 October 2020, Revised: 19 May 2021, Accepted: 23 May 2021

\begin{abstract}
Men and women may experience depression very differently. The previous studies found that women expressed emotional distress mean that they were more likely to be diagnosed with depression, while some men with depression tried to hide their emotions, so causing them to have delayed treatment. This qualitative research aimed to study the perception and management of depressive symptoms in Thai males before receiving treatment with psychiatric specialists. The participants who were diagnosed major depressive disorders and dysthymic disorders were 23 men by purposive sampling was used. Data were collected through in-depth interviews by a semi-structured interview, which was based on literature review and it was verified by advisor, the second author who experienced of qualitative study. Analyzed by content analysis based on the process of Colaizzi. The finding revealed that the perception and management of depressive symptoms were divided into 3 phases. Phrase 1 was the perception of abnormal symptoms affecting daily activities but an attempt to hide it and struggling for a normal life. Phrase 2 showed that Thai men's perception of symptoms were thinking too much, ruminative thought causing suffering, and then trying to release the suffering and control ruminative thought. Phrase 3 was the perception of loneliness, hopelessness, suicidal thoughts, and then trying to seek help. Healthcare providers can use the findings as information for developing screening tools specific to Thai men. The information can also be used to develop psycho-education tools for close ones or family members to recognize and understand early symptoms so that care, assistance and access to treatment can be provided before severe depression is developed. The research was only carried out in 1 area, the results could not be generalized, besides that it could have multiple meanings or be biased if it was carried out in other areas with different cultures.
\end{abstract}

Keywords: Perceptions, Management, Depression, Men, Northeast Thailand

\section{Introduction}

Major depressive disorder is "a silent health disaster" that is found in all countries around the world. The World Health Organization has estimated that there are more than 322 million people with depression worldwide, amounting to $4.4 \%$ of the world's population [1]. Moreover, a report from WHO suggests that depression will be the single biggest cause of ill health in the world by 2030 [2]. In Thailand, according to a 2020 report of depressed patients (not differentiated by gender), $2.08 \%$ of the population aged 15 and over had depression [3]. According to epidemiological evidence, the proportion of women with major depressive disorder is higher than men in a ratio of 3 to 2 [4,5]. However, depression in men is currently attracting increasing interest because men who are depressed may appear to be angry, aggressive, and have substance abuse issues instead of presenting typical depressive symptoms such as sadness [6-11]. Moreover, their families, close friends, and some health care providers may not recognize the anger or aggressiveness as depression symptoms or may misdiagnose the behavior [8], which is consistent with a previous study by Borowsky et al. [12] found that health care providers are less likely to diagnose mental illness in men than women, because men are less likely than women to recognize, talk about, and seek treatment for depression $[11,13,14]$. 
Men and women may express symptoms of depression very differently. Previous studies found that women expressed emotional distress which meant that they were more likely to be diagnosed with depression, while some men with depression tried to hide their emotions $[6,9,11,15]$, so women were more likely than men to receive treatment [16]. A study in western countries such as United States, Sweden and Australia, etc., found that traditional image of masculinity related to depression and lower well-being in men [17,18] and reduced willingness to seek help [18,19]. Traditional masculinity means that men must be strong and not cry. They must not show weakness and know how to control their emotions [6,20-22]. Moreover, a previous study found that men's perceptions of depression related to weakness and a loss of self-control [11,14,23], as opposed to the masculine identity that society expects. Therefore, when depression occurs, men try to conceal and hide their emotions, known as "masked" or "hidden" depression [6,9,11,15], which may cause a delay in diagnosis and treatment [16]. Men may deal with depression by enduring it and trying to hide their emotions and feelings. They also drank alcohol to relieve stress and avoid seeking help because they believed that a man must be able to look after and help himself $[6,24,25]$, which may be a contributing factor to the high prevalence of alcohol and substance abuse in men [26].

A study also found that men with depression reported that receiving treatment with a specialist was the last option they would choose [6,25] and looking for help from an expert was an expression of weakness [25]. They also did not believe that psychiatrists can help them and did not want to take medicine [16]. Many men suffered from depression until becoming severely depressed, sometimes leading to suicide $[9,27,28]$.

As seen by the previous discussion, major depressive disorders in men are complex. A review of literature from 2000 onwards shows that some qualitative research has been conducted to understand the experience of men with depression or major depressive disorder. However, no study was found that was conducted to clearly understand the thought processes and feelings of men with major depressive disorder from their subjective recognition depression and how to deal with the symptoms prior to undergoing treatment.

Therefore, this study was conducted to understand the perception and management of depressive symptoms in Thai working age males before receiving treatment with psychiatric specialists. The findings will be beneficial for health officers as a basis for screening and finding symptoms at the initial stages of depression in men to promote early treatment for men with major depressive disorders. It will also help close friends or family members to know and understand the symptom that occur in the initial stages of men with major depressive disorder so that care, assistance and access to treatment can be provided before severe depression is developed.

\section{Materials and methods}

\section{Study design}

An exploratory, descriptive qualitative design was used. During semi-structured, in-depth interviews, 23 Thai men who were diagnosed with major depressive disorder (MDD) and dysthymic disorders talked about their experiences with depression and explored the perception and management of depressive symptoms.

\section{Participants and data collection}

Following university ethics approval, psychiatric nurses working at the outpatient departments of the hospitals chosen for the research, invited 25 young men to participate in the study. Twenty-three men agreed to participate and signed informed consent forms. Two men declined to participate in this study. Those who willing to participate in this study were asked to provide contact information so that researchers could reach them to review eligibility and discuss next steps. Participants were men of working age who were diagnosed with major depressive disorder (MDD) and dysthymic disorders. Participants were selected by a purposive sampling method. Inclusion criteria included: 1) males aged between 18 - 59 years, 2) born in the province of northeastern Thailand, 3) not having severe mental symptoms and, 4) willing to participate in this study.

The 23 participants differed in age, education level, occupations, and duration of illness with depression. Data were collected through in-depth, semi-structured interviews by the first researcher, which was based on literature review and it was verified by an advisor who experienced of qualitative study interviews (The sample interview questions are shown in Table 1). The duration of each interview was 60 to $90 \mathrm{~min}$. Each informant was interviewed 1 - 3 times until data saturation was reached. Verbatim transcription was done with the data obtained from the interviews. 
Table 1 Examples of semi-structured interview questions.

\section{Examples of semi-structured interview questions}

1) What was the first depressive symptom that you recognized?

2) At the moment your perception of a depressive symptom occurred, what did you think happened to you?

3) Could you please talk about how you managed this symptom and what was the result?

4) Why did you decide to seek help?

\section{Data analysis}

Content analysis was performed using the 7 steps of Colaizzi's method [29] including: 1) read and reread the obtained data for understanding before analyzing and coding the data, 2) code and categorize the data pertaining to the study issues, 3) define the meaning of study issues obtaining from phrases, sentences, or significant statements, 4) cluster the similar meanings and determine a theme, 5) extensively illustrate the meaning of study issues, 6) describe all the significant structures of the study issues, and 7) discuss the results with an advisor to validate the descriptions for formulating the meaning of the extracted data, and repeat reading the description together if there is incongruence.

\section{Quality of data}

To ensure the trustworthiness of the study, triangulation method was employed, including sources of informants, data collection methods, field notes and observations and memory recording. All data analysis was then further cross-checked and closely reviewed by the advisor. Data or results were returned to participants to check for accuracy and resonance with their experiences. Seventeen participants were consulted because some participants were unavailable for a variety of reasons.

\section{Ethical considerations}

This study was approved by the University Research Ethics Committee (HE611531). All participants were given written information about the study. Participants' names and identifying details were removed from records before analysis.

\section{Results and discussion}

Participant's characteristics

There were 23 males of working age who mostly were diagnosed with major depressive disorder within 1 year of 14 patients, out of which, 5 patients had dysthymic disorders. The mean age was 39.9 years (range, 18 - 59 years). All were born in a province of northeastern Thailand; most were Buddhist and graduated with a bachelor's degree or higher. Roughly half were married, and half were single. The participants had a variety of occupations, including government officer, farmer or self-employed. A depression assessment using the 9Q found that mostly participants were no longer depressed, and mostly they had no comorbid diseases (details in Table 2). The cultural background of the participants was reported in the research article: "Working-age Northeastern Thai males' perception of causes of depression", published on Journal of Thailand Nursing and Midwifery Council [30].

Table 2 Demographic characteristics of participants.

\begin{tabular}{|c|c|c|c|c|c|}
\hline Characteristic & number & Characteristic & number & Characteristic & number \\
\hline $\begin{array}{c}\text { Age } 18-59 \\
\text { Mean }=39.90 \\
\mathrm{sd}=13.8\end{array}$ & 23 & Level of education & & $\begin{array}{l}\text { Duration of } \\
\text { depression }\end{array}$ & \\
\hline Religion & & Primary school & 7 & $<6$ months & 8 \\
\hline Buddhism & 22 & Secondary school & 6 & 6 months - 1 year & 6 \\
\hline Christianity & 1 & $\begin{array}{c}\text { Bachelor's or } \\
\text { Master's degree }\end{array}$ & 10 & $>1-2$ years & 3 \\
\hline
\end{tabular}




\begin{tabular}{cccccc}
\hline Characteristic & number & Characteristic & number & Characteristic & number \\
\hline Marital status & & Occupation & & 3 years & 1 \\
\hline Single & 10 & Unemployed & 2 & $8-10$ years & 3 \\
\hline Married & 10 & Farmer/self employed & 7 & $11-12$ years & 2 \\
\hline $\begin{array}{c}\text { Widow/Divorce } \\
\text { Household } \\
\text { menbers }\end{array}$ & 3 & Goverment officer & 8 & Comorbid disease & 18 \\
\hline$<3$ persons & 11 & Company employee & 2 & No & 5 \\
\hline$>3$ persons & 12 & Student & 2 & Pepression & assessment (9Q) \\
\hline & & Clergy & 1 & No longer depressed & 13 \\
\hline
\end{tabular}

Depressive symptoms perception and management

The 2 major themes identified in interviews were perception and management of depressive symptoms. Each of these themes consisted of 3 phases of depressive symptom perception and management, including: Phase 1) perception of depressive symptoms affecting daily activities accompanied by trying to hide, endure, and move on, phase 2) perceived symptoms of overthinking, suffering, and trying to ease the suffering and control ruminative thought; phase 3) perception of loneliness, and wanting to die, then trying to seek help (details in Table 3).

Table 3 Numbers of depression symptom perception and management in phase 1, 2 and 3.

\begin{tabular}{lclc}
\hline \multicolumn{1}{c}{ Symptom perception } & number & \multicolumn{1}{c}{ Symptom management } & number \\
\hline $\begin{array}{l}\text { Phase 1: Depressive symptoms } \\
\text { affecting daily activity }\end{array}$ & 15 & $\begin{array}{l}\text { Masculinity influences symptoms } \\
\text { management }\end{array}$ & 15 \\
\hline $\begin{array}{l}\text { Body sleep but brain awake all the } \\
\text { time }\end{array}$ & 13 & Use addictive substance & 10 \\
\hline Bad mood/bad temper & 7 & Getting out of stressful situations & 6 \\
\hline Fatigue & 5 & Try to tolerate and move on & 5 \\
\hline $\begin{array}{l}\text { Lack of concentration on work and } \\
\text { work inefficiently }\end{array}$ & 10 & $\begin{array}{l}\text { Trying to release the suffering and } \\
\text { control ruminative thought }\end{array}$ & Seeking information related depression \\
\hline $\begin{array}{l}\text { Phase 2: Overthinking and wanting } \\
\text { to be alone }\end{array}$ & 7 & Ease the suffering & 12 \\
\hline Ruminative thought & 7 & Practice meditation & 7 \\
\hline Wanting to be alone & & Trying to seek help & 20 \\
\hline Feeling bored & 11 & $\begin{array}{l}\text { Seeking help from the health } \\
\text { professional }\end{array}$ \\
\hline Phase 3: Lonely and wanting to die & 7 & Seeking help from close one & 5 \\
\hline \begin{tabular}{l} 
Feeling sad and lonely \\
\hline Want to die/self-harm
\end{tabular}
\end{tabular}




\section{Phase 1: Perception of depressive symptoms affecting daily activities but trying to hide, endure, and move on}

During this phase, 21 participants did not know that they were suffering from depression. They did perceive that there was something wrong with themselves.

Depressive symptoms affecting daily activity

Twenty-one participants perceived an occurrence of depressive symptoms because these symptoms had affected their activities of daily living. The initial symptoms of depression that they experienced were physical symptoms, including a feeling that their body was asleep, but their brain was awake all the time, a bad mood/bad temper, fatigue, a lack of concentration at work or working inefficiently, headache/backache, nervousness, and a shortness of breath. The 3 most frequently reported depressive symptoms were as follows:

Body asleep but brain awake all the time

Fifteen participants reported that initial depressive symptoms were sleeplessness or intermittent sleep; nightmares resulting in light sleep; or often waking up in the middle of the night but not able to get back to sleep. Some participants reported that their body slept but their brain was awake, resulting in exhaustion, as a participant addressed.

"I couldn't sleep, it differed from other times that I couldn't sleep because I kept thinking the same thing over and over again, it seemed like my body slept but my brain was still working all the time. I told the doctor that it was the first time that I felt like that I was dreaming but I wasn't. I freaked out. I woke up frightened and couldn't get back to sleep.” ........ ID_03_05_34 years old_MDD 1 year 3 months_1/8

\section{Bad mood/bad temper}

Thirteen participants reported that their symptoms were being cranky, in a bad mood, or a bad temper. Some participants stated that they could not control their emotions, leading to quarrels with coworkers, bosses, or girlfriends. Most married men said that if their wife said or did something that did not please them, they felt irritable and had a bad temper, and sometimes they wanted to hurt her.

"When my wife raised her voice or said something bad to me it made me angrier. It was to the point that I wanted to kill her because of the way she talked to me... it seemed like I was an insane person. Previously, I had never had this kind of feeling. This feeling was with me for long, long time; my oldest child would not come to see me because he was scared of me. "........ ID_04_01_55 years old MDD 3 months_1/2

\section{Fatigue}

Seven participants reported that the initial depressive symptom occurring often was being tired/exhausted. One participant said that he was tired, exhausted, suffocated; it seemed like he was going to die.

"At that time when this symptom occurred, I thought it was achy, but I had no energy and felt so moody, I could not control my emotions. My heart was beating so strong, and it seemed like... I was in shock and so weak." ....... ID_04_03_29 years_MDD 2 months_1/1

Masculinity influences symptoms management

Twenty-two participants perceived that their initial depressive symptom was stress, therefore they tried to manage their stress on their own before seeking help or telling anybody about their symptom. Participants said that because of their idea of masculinity, they had to hide their feelings or emotions with various measures according to individual's personality, including: 1) tolerating and moving on, 2) using addictive substances, 3 ) getting out of stressful situations and 4) engaging in a variety of activities.

\section{Tolerating and moving on}

Five participants reported that they had an introvert personality. They had few friends and did not socialize much. Usually, they did not like to drink alcohol. When they perceived their symptom occurring, they tried to hide their feelings or emotions without telling anyone. They believed that men would not express their feelings or emotions to anyone. Some participants tried to keep their emotions and suffering to themselves for long time. They tried to tolerate it and move on until more depressive symptoms developed, as an example below. 
"Because I am a man, I don't say how I felt. What I did, I just kept it with me all the times...kept it with me for so long until I felt I couldn't take it anymore...I tried to carry everything." ........ID_01_01_20 years old_MDD 6 months_1/5

Using addictive substances

Ten participants reported that they were usually quiet and untalkative. They tried to hide their feelings, emotions, suffering, and uneasiness within themselves until they could not tolerate it. Then they turned to alcohol to relieve their stress and suffering. They believed that when they got drunk it would help them forget their suffering. Three informants used marijuana (cannabis); they believed that marijuana made them amused and relaxed, in a good mood, happy, and able to enjoy chatting with friends. They felt more relaxed when using addictive substances.

"It has been in Thai society for a long time. I believe that every man, when being stressed or feeling uncomfortable, has to drink alcohol. Or some may use narcotics and become addicted." ............ ID_03_06_38years_MDD3 months_1/3

"Marijuana, when I am very stressful, I use it as it is enjoyable. It is OK. It is better than drinking alcohol. It's really relaxing." ID_02_04_32 ปี_MDD 3 months_1/8

\section{Getting out of stressful situations}

Six participants said that their characteristics included impatience, irritability, anger, impulsive thinking, and hasty decision making. When they faced challenging situations, they often failed to control their emotions, so they frequently had arguments with family members. Later they tried to get out of stressful situations in order to reduce their irritability.

"When my wife scolds our grandchildren, I am usually irritated. As a man, I have to control my emotions. I will walk out and grab the car keys and drive to see friends for an hour or two. When I am in a good mood, I will go back home. That's it. Others may think I am crazy, but I'm not." ID_03_01_48years_MDD3 months_1/3

Various activities to relieve stress

Fifteen participants stated that they tried to do different activities to make themselves feel relaxed and relieve stress. Some watched movies and listened to music to make themselves feel entertained so that they did not think about suffering or uneasiness. Some drove around, read books, did exercise, and hung out and sang karaoke with friends.

"I kept driving alone. That day, I fell asleep, so the car ran off the road. Fortunately, it didn't damage anything. "............. ID_03_06_38 years_MDD2 months_1/9

Phase 2: Perceived symptoms of overthinking, suffering, and trying to ease the suffering and to control ruminative thought

After participants had tried to hide their emotions and manage themselves with various activities mentioned above, the symptoms did not improve and began to get worse with overwhelming thoughts and separation from friends and social activities, therefore entering phase 2 which consists of 2 themes, 1) Depression symptom perception: Overthinking and wanting to be alone and 2) Symptoms management: Trying to ease the suffering and control ruminative thought.

Depression symptom perception: Overthinking and wanting to be alone

In this phase participants had symptoms of overthinking, did not want to socialize, and did not want to do anything. They also had negative thoughts, decreased self-esteem and boredom, and lacked hope and encouragement. The 3 most reported symptoms during this phrase were as follows.

\section{Ruminative thought}

Ten participants said that the symptoms they perceived to be more severe were overthinking and ruminative thought. They kept thinking about the things that happened in the past, which made the symptoms worse.

"Overthink; I would think about only the past events. Always think about what mistakes I have made that made me face this situation. I keep thinking about old matters. Even at the present, I am still thinking about it..... until it becomes ruminative thoughts. I think about the old things and think of my children and wife... until I feel bored and depressed." ID_02_05_52 years_MDD 9 years_1/2 
Wanting to be alone

Seven participants reported that the symptom they observed later was a desire to withdraw. They did not want to socialize. They wanted to be alone and live in their own world. Sometimes, when others talked to them, they heard it, but they did not want to respond.

'I don't want to talk to anyone. I want to be alone. When someone talks to me, I hear it but I don't want to talk to them. I want to be alone, but I don't want to commit suicide. I think it's not normal because when others talk to me why I don't want to talk to them .... I have had this symptom for about 1 year." ............ID_03_04_20 years_MDD1 year_1/1

\section{Feeling bored}

Seven participants reported that later symptoms were boredom, and not wanting to do anything.

"I feel bored. Doing things is not fun as before. In the past, I liked listening to music in the morning, but now when listening to it, it is not fun. Planting trees is not also fun. I'm bored." D_03_08_31 years_MDD1 year 2 months_1/7

Symptoms management: Trying to ease suffering and control ruminative thought

In this phase, participants began to manage their suffering differently from the earliest stage, namely attempting to ease the suffering, practicing meditation and seeking information. It was found that participants had different methods of self-care at this stage varying according to age, thoughts, beliefs and family contexts.

\section{Ease the suffering}

Eleven participants stated that they tried to ease their suffering in various ways according to their age and family contexts, including talking about their suffering with people whom they trusted and through Facebook.

1) Sharing with a trusted person

Four participants ages 23 - 31years old provided the information that they were close to their mothers and had warm, loving families. When they felt uncomfortable, most of them talked to their mothers. After talking with their mothers, it helped them feel more relaxed.

"Sitting and talking with my mother about how my job was helped. When talking about my supervisor, I suddenly felt angry. It was like I kept it and waited for the time to be released. When I released it out, I felt relieved talking to my mother" ........... D_03_08_31 years_MDD1 year 2 months_1/7

\section{2) Using Facebook}

Seven participants who were in early adulthood and completed bachelor's degrees or were currently studying and had IT and social media skills stated that when they felt uneasy, they released their emotions by posting on Facebook or using images to convey emotions, feelings, and distress to help them feel somewhat better.

"When I had no way out, I posted on Facebook. There were only depressing posts. Self-blaming implied that I was sad and it made me feel better." months_1/6

\section{Practicing meditation}

Buddhist practice through meditation to calm the mind was described as a method that 7 informants tried to use when they felt distressed by overthinking. They provided information that in the past they were ordained as monks and learned to practice meditation. Most of them explained that it helped the symptoms of overthinking to pass. Some informants said that meditation helps them try to focus, concentrate, calm their mood, and forgot their bad experiences including painful emotions.

"Throughout my life, my parents have always taken me to the temple. I also used to go to a temple to meditate. So, I think that meditation is the best. If we meditate regularly, we can gather our minds at 1 point. It will make you think less. The brain will be empty and it will make you relaxing and good feeling." ID_03_08_31 years_MDD1 year 2 months_1/6

\section{Seeking information related to depression}

Twelve participants were in early adulthood, with computer knowledge and skills. They tried to take care of themselves in various ways, as mentioned earlier. However, the symptoms did not improve until 
the people around them, such as friends, wives and girlfriends, began to reflect that they were not the same and may have mental health problems because they were easily irritated and angry. They were curious whether these observations were correct, so they began to seek information from various sources. Most of them searched for information from the internet, books, and other people, including friends and health officers.

"I searched on the website of the Department of Mental Health. It is stated that having depression does not mean that you are crazy. It is treatable and can be cured. The information I was looking at made me dare to get to treatment.... But if you do not receive treatment, you will be crazy, eventually.". ID_02_03_32 years_MDD7 months_1/9

\section{Phase 3: Perception of aloneness and wanting to die, and trying to seek help}

At first participants tried to manage depressive symptoms by themselves. But the symptoms did not improve because they had overwhelming thoughts and felt isolated. Eventually the symptoms became so severe that they were having suicidal thoughts or thoughts of self-harm. In this period their depression was severe. They felt so lonely, hopeless and suicidal that they could not bear it. Therefore, they tried to seek help, as detailed below.

Depression symptom perception: Lonely and wanting to die

The participants said that after they had tried to ignore the symptoms, manage it themselves, and ease the suffering, the symptoms did not improve. Instead, they felt more depressed, to the point of it being unbearable. They felt sad, lonely and discouraged and did not want to live. They also thought of dying and hurting themselves.

\section{Feeling sad and lonely}

Eleven participants who were both in early adulthood and middle adulthood stated that the abnormal symptoms were the feelings of sadness, loneliness and discouragement. These symptoms usually occurred in the evening without them knowing the causes. They felt like they were alone and no one understood their feelings.

"I feel that I am the only person in the world in that area although it is a village, and my mother's house is located nearby. I live in the building that I rent with my girlfriend, but I wonder why I feel like being alone in this world. ", ID_02_02_24 years_MDD8 years_1/12

\section{Wanting to dielself-harm}

Seven participants who were both in early adulthood and middle adulthood said that the symptoms they could not bear were the feelings of being discouraged and wanting to die. Some stated that they had planned to commit suicide by gunshot or hanging. Others reported that they had committed self-harm.

"I didn't know what to live for. The idea of self-harm began to emerge. The first time I had this feeling was when I was in Year 2, second semester. The first time I tried to get hit by a car, but the car did not hit me. Nothing happened. Then I continued studying. For the second time and the third time, I had to be admitted to the hospital. I slit my arms. "............ID_01_01_20 years_MDD6 months_1/3

Symptoms management: Trying to seek help

After the participants perceived that the symptoms were more severe, they realized they could not manage themselves and began to seek help. Initially, they began to seek help from close ones in the family, especially from wives and mothers but as the symptoms did not improve, they sought help from health professionals.

Seeking help from close ones

Five participants were those who lived in a family with good relationships among the family members. They began to ask for help from the people they trusted in the family first, namely wives and mothers. Most of family believed that the abnormal symptoms might be caused by the supernatural. Therefore, they relied on black magic, such as consulting fortunetellers, changing horoscopes for a better life, making a votive offering, dancing to pay respects to ghost spirits, being sprinkled with holy water, and chanting.

"According to a rural belief, depression is caused by being possessed by a ghost. Simply speaking, it is an illness caused by a ghost. So, we have to see a fortuneteller... The fortuneteller told us to decorate a Buddha shelf at home to worship and do offerings to ...My wife believes in this. She believes in ghosts." .........ID_03_03_52 years_MDD 7 months_1/24 


\section{Seeking help from the health professional}

Twenty participants said that at a certain point they felt they could not bear the symptoms, particularly physical symptoms such as insomnia, tiredness, fatigue and palpitations. Therefore, the family members started looking for medical help. Most of them were checked by physicians, but the causes of the abnormalities were not found.

"My mother took me to see a doctor at the clinic). After the examination, nothing was found. I went to see a doctor at the hospital. Again, nothing was found. I also went to the Heart Center and had a check, but there was nothing wrong. ....... It lasted for almost 1 month at that time." .......... ID_02_03_32 years_MDD7 months_1/1

\section{Discussion}

The results of this study revealed that the symptoms of depression among men in Thai society are not different from those in Western societies, where previous studies found that men with depression tried to hide their emotions and they are less likely than women to seek treatment $[6,8,9,11,15,25,31]$. Because Thai society is still male-dominated [32], most men adhere to traditional masculinity which believes that men must be strong and enduring. They must be the leaders, must not cry and show weakness, must know how to control their emotions and be able to take care of themselves [33]. Therefore, many participants with major depressive disorders suffered from depression for years, especially those saying that they did not know about depression and symptoms of depression. They thought that the symptoms that occurred were stress, so they tried to manage stress by themselves. In addition, participants managed their depressive symptoms in a similar way as found in previous studies in Western countries. A systematic review and meta-synthesis of qualitative studies [17] found that traditional masculinity influenced how men managed depressive symptoms. They employed avoidance coping strategies, such as focusing extremely on work and/or sports, hanging out with friends or letting go and using drugs and alcohol [6,7,9-11,17] or smoking in an attempt to cope or alleviate the stress [8]. On the other hand, some qualitative studies found that men coped with stress and depression through spiritual and religious practices such as church attendance or their relationship with God, consulting clergy or fellow church members $[9,14]$ but their depressive symptoms often returned or increased $[22,34]$.

The management of depressive symptoms by Thai males has some differences with management of depressive symptoms by males in Western cultures because of differing cultural and religious beliefs. The self-management of symptoms of participants with major depressive disorder was linked with ideas and beliefs in Thai society, culture and religious practices. This study found that those who practiced meditation-based religious principles were mostly middle-aged men who were ordained as monks and learned to practice meditation. Ordination is a value of Thai society. Parents believe that when they pass away, they will go to heaven because of the merit gained from their son's ordination. At the same time, many men believe that ordination is regarded as the greatest merit that they can repay their parents. Most men in Thai society have experienced life as a monk at some time in their life. They have learned and been close to the way of life of the Sangha (monk) society and learned mental training and meditation. Therefore, when they overthink and have ruminative thoughts, they use meditation to take care of themselves.

Moreover, the participants who had major depressive disorders tried to manage the symptoms by themselves using various methods, most of which were based on family beliefs, such as making merit, offering food and necessary things to Buddhist monks, being sprinkled with holy water, chanting, feeding ghosts and ancestors' spirits and getting ordained. These findings were consistent with a previous study by Rungreankulkij et al. [35] which found that Thai male participants with depression started seeking help using superstition-based treatment, such as being sprinkled with holy water and being treated by Modhum (the person having folk medicine knowledge) which were based on family beliefs [35]. This may be because when men are in psychological distress, they rarely seek help from others, including mental health professionals, because seeking help that related to weakness $[11,14,23]$ but they are willing seeking advice from their family members [8], especially their wives. The treatment based on ideas, beliefs, and faith of the individual is considered a spiritual healing.

Men have also defined the meaning of depression as a spiritual injury [10], which is the emotional suffering that affects spirituality, causing decreased self-worth and self-esteem, lack of confidence [28], fear and depression [9] until it leads to spiritual suffering, ultimately resulting in suicidal ideation. Therefore, practicing meditation care is a spiritual remedy making people become strong by helping people to accept their experiences including painful emotions, rather than react to them with avoidance. Many people who practice mindfulness find that they are less likely to get caught up in worries about the 
future or regrets over the past [36]. Most participants stated that after they did meditation, they had a lot of control over their thinking, stayed focused on the present, reduced negative emotions, and hoped of improving their symptoms.

This study supports the theory of some scholars who suspect that depression is a cultural illness [37], especially adherence to traditional masculinity as it relates to depression symptoms and management $[6,8,20-22]$, so understanding and acceptance within these societies depends on class, gender and culture $[9,38]$. Masculinity is shaped by society and culture in that context. Masculinity changes depending on the context of society, culture, ideas, beliefs and social and cultural values, which evolve over time. Men in Western cultural societies and men in Thai society may have different ideas, beliefs, and values towards masculinity. So, this study found that participants had some different approaches to the management of depressive symptoms compared to men in Western cultures.

\section{Conclusions}

The results of the study revealed interesting issues about the perception of depression and the path of participants before receiving treatment from psychiatric specialists. The path can be divided into 3 phases according to the duration and the perceived severity of the symptoms. Phase 1: Perception of depressive symptoms affecting daily activities but trying to hide, endure, and move on. Phase 2: Perceived symptoms of overthinking, suffering, and trying to ease the suffering and to control ruminative thought. Phase 3: Perception of aloneness and wanting to die and trying to seek help.

\section{Relevance for clinical practice}

The findings can be used by health officials as basic information for screening and assessment of atrisk groups of men. Behaviors such as acting out, aggressiveness, use of addictive substances, a lack of concentration on work can be indicators of depression. The findings can also be used to provide education to the families of depressed men, teaching them about the depressive symptoms that may occur in men.

\section{Study limitations}

The study was conducted only in Khon Kaen province and nearby areas located in the northeastern region of Thailand. Therefore, the use of this research should account for the context of the geographic area. The results could not be generalized; other geographic areas may have cultures that are different enough to make the findings here unreliable.

\section{Acknowledgements}

This article is a part of dissertation for a $\mathrm{PhD}$ in Nursing. I would like to express sincere gratitude to all the participants for taking time to provide useful information, and to the Center for Research and Training on Gender and Women's Health, Faculty of Nursing, Khon Kaen University, Thailand for research fund support. All authors declare that they have no conflict of interest.

\section{References}

[1] World Health Organization. WHO on suicide prevention, Available at: http://www.who.int/mental_health/prevention/suicide/suicideprevent/en/, accessed August 2017.

[2] Asian News International. World mental health day: Depression to be the biggest cause of ill health by 2030, says WHO, Available at: https:/www.indiatoday.in/education-today/gk-currentaffairs/story/world-mental-health-day-depression-suicide-prevention-who-1607866-2019-10-10, accessed September 2020.

[3] Depression Research and Information Center. Report on access to depressive patients, Available at: http://www.thaidepression.com/www/report/main_report/, accessed May 2020.

[4] World Federation for Mental Health. Depression: A global crisis world mental health day, Available at: https://www.who.int/mental_health/management/depression/wfmh_paper_depression_wmhd 2012.pdf, accessed August 2017.

[5] P Kittirattanapaiboon, $\mathrm{N}$ Tantirangsee, $\mathrm{W}$ Chutha, A Tanaree, $\mathrm{P}$ Kwansanit and $\mathrm{S}$ Assanangkornchai. Prevalence of mental disorders and mental health problems: Thai national mental health survey 2013. J. Ment. Health Thai 2017; 25, 1-19. 
[6] AB Rochlen, DA Paterniti, RM Epstein, P Duberstein, L Willeford and RL Kravitz. Barriers in diagnosing and treating men with depression: A focus group report. Am. J. Men's Health 2010; 4, 167-75.

[7] JL Oliffe, PM Galdas, CS Han and MT Kelly. Faux masculinities among college men who experience depression. Health (London) 2023; 17, 75-92.

[8] Kendrick, NLR Anderson and B Moore. Perceptions of depression among young African American men. Fam. Commun. Health 2007; 30, 6373.

[9] K Bryant. 2008, The impact of depression on middle-aged African American men: A grounded theory approach. Ph. D. Dissertation. Azusa Pacific University, Azusa, California.

[10] D Christopher, JM Greenfeld, ST Greenberg, SJ Shepard, SV Cochran and JT Haley. A qualitative investigation of depression in men. Psychol. Men Masculin. 2009; 10, 302-13.

[11] A Brown, U Scales, W Beever, B Rickards, K Rowley and K O’Dea. Exploring the expression of depression and distress in aboriginal men in central Australia: A qualitative study. BMC Psychiatr. 2012; $12,97$.

[12] SJ Borowsky, LV Rubenstein, LS Meredith LS, P Camp, M Jackson-Triche and KB wells. Who is at risk of nondetection of mental health problems in primary care? J. Gen. Intern. Med. 2000; 15, $381-8$.

[13] W Affleck, V Carmichael and R Whitley. Men's mental health: Social determinants and implications for services. Can. J. Psychiatr. 2018; 63, 581-9.

[14] DL Hudson, J Eaton, A Banks, W Sewell and H Neighbors. "Down in the sewers": Perceptions of depression and depression care among African American men. Am. J. Men's Health 2018; 12, 12637.

[15] ME Addis. Gender and depression in men. Clin. Psychol. Sci. Prac. 2008; 15, 153-68.

[16] J Angst, A Gamma, M Gastpar, JP Lépine, J Mendlewicz and A Tylee. Gender differences in depression. Eur. Arch. Psychiatr. Clin. Neurosci. 2002; 252, 201-9.

[17] S Krumm, C Checchia, M Koesters, R Kilian and T Becker. Men's Views on depression: A systematic review and metasynthesis of qualitative research. Psychopathology 2017; 50, 107-24.

[18] RF Levant, DG Stefanov, TJ Rankin, MJ Halter, C Mellinger and CM Williams. Moderated path analysis of the relationships between masculinity and men's attitudes toward seeking psychological help. J. Couns. Psychol. 2013; 60, 392-406.

[19] ZE Seidler, AJ Dawes, SM Rice, JL Oliffe and HM Dhillon. The role of masculinity in men's helpseeking for depression: A systematic review. Clin. Psychol. Rev. 2016; 49, 106-18.

[20] L Lynch, M Long and A Moorhead. Young men, help-seeking, and mental health services: Exploring barriers and solutions. Am. J. Men's Health 2018; 12, 138-49.

[21] I Sagar-Ouriaghli, E Godfrey, L Bridge, L Meade and JL Brown. Improving mental health service utilization among men: a systematic review and synthesis of behavior change techniques within interventions targeting help-seeking. Am. J. Men's Health 2019; 13, 138-49.

[22] A Keohane and N Richardson. Negotiating gender norms to support men in psychological distress. Am. J. Men's Health 2018; 12, 160-71.

[23] B Spillman. 2006, Men's experiences of depression: A phenomenological investigation. Ph. D. Dissertations. University of Tennessee, Knoxville, USA.

[24] R Rungreankulkij, I Kotnara, W Kittiwatanapaisan and S Arunpongpaisal. Loss of control: experiences of depression in Thai men. Walailak J. Sci. Tech. 2019; 16, 265-74.

[25] JL Johnson, JL Oliffe, MT Kelly, P Galdas and JS Ogrodniczuk. Men's discourses of help-seeking in the context of depression. Sociol. Health Illness 2012; 34, 345-61.

[26] H Ritchie and M Roser. Mental health, Available at: from https://ourworldindata.org/mental-health, accessed August 2018.

[27] S Brownhill, K Wilhelm, L Barclay and V Schmied. 'Big build': Hidden depression in men. Aust. New Zeal. J. Psychiatr. 2005; 39, 921-31.

[28] U Danielsson and EE Johansson. Beyond weeping and crying: A gender analysis of expressions of depression. Scand. J. Prim. Health Care 2005; 23, 171-7.

[29] GA Shosha. Employment of colaizzi's strategy in descriptive phenomenology: A reflection of a researcher. Eur. Sci. J. 2010; 8, 31-43.

[30] N Kaewjanta and S Rungreangkulki. Working-age northeastern Thai males' perception of causes of depression. J. Thai. Nurs. Midwifery Counc. 2021; 35, 18-34.

[31] S Rungreangkulkij. Depression in men: Gender lens. Songklanagarind J. Nurs. 2017; 37, 158-65.

[32] N Rodsup. Patriarchy: The reflection of the inequality between male and female in Asian society. $J$. Res. Dev. Buriram Rajabhat Univ. 2012; 4, 30-46. 
[33] N Kaewjanta. 2020, The adaptation process of Isan, Thai men with major depression: A grounded theory approach. Ph. D. Dissertation. Khon Kaen University, Khon Kaen, Thailand.

[34] EL Whittle, AS Fogarty, S Tugendrajch, MJ Player, H Christensen, K Wilhelm, D Hadzi-Pavlovic and J Proudfoot. Men, depression, and coping: Are we on the right path. Psychol. Men Masculin. $2015 ; \mathbf{1 6}, 426-38$.

[35] S Rungreankulkij, I Kotnara, W Kittiwatanapaisan and S Arunpongpaisal. Depressive disorder: Gender analysis of depression. J. Nurs. Sci. Health. 2012; 35, 21-30.

[36] Ohio University. Mindfulness, Available at: https://www.ohio.edu/studentaffairs/counseling/ mindfulness, accessed October 2020.

[37] C Dowrick. Depression as a culture-bound syndrome: implications for primary care. Brit. J. Gen. Pract. 2013; 63, 229-30.

[38] ZF Khalaf, WY Low, B Ghorbani and EM Khoei. Perception of masculinity amongst young Malaysian men: A qualitative study of university students. BMC Publ. Health 2013; 13, 1062-70. 